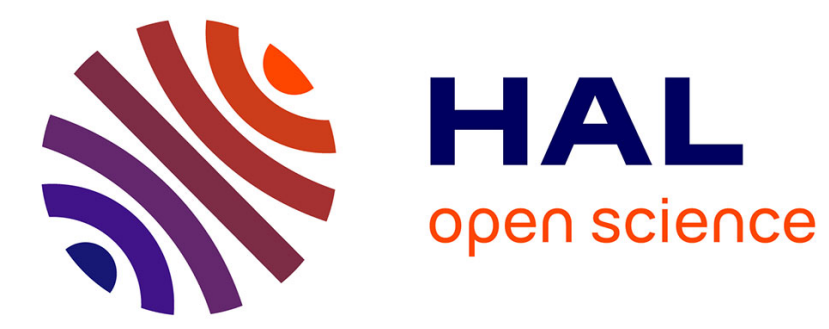

\title{
La source et l'écart
}

Houria Abdelouahed

\section{To cite this version:}

Houria Abdelouahed. La source et l'écart. Cliniques méditerranéennes, 2006, 1 (73), pp.115 - 127. 10.3917/cm.073.0115 . hal-01508588

\section{HAL Id: hal-01508588 \\ https://hal.science/hal-01508588}

Submitted on 19 Jun 2017

HAL is a multi-disciplinary open access archive for the deposit and dissemination of scientific research documents, whether they are published or not. The documents may come from teaching and research institutions in France or abroad, or from public or private research centers.
L'archive ouverte pluridisciplinaire HAL, est destinée au dépôt et à la diffusion de documents scientifiques de niveau recherche, publiés ou non, émanant des établissements d'enseignement et de recherche français ou étrangers, des laboratoires publics ou privés. 


\section{Houriya Abdelouahed}

\section{La source et l'écart}

«Il n'y a pas de visage sans une âme. » Aristote, De la Génération des animaux.

«Il faudrait donc que nous entendions déjà le sens du mot origine quand il désigne toute autre chose que le jaillissement d'un cours d'eau, pour accéder à ce qui pourtant nous était proposé comme le propre sens de la source. » Jacques Derrida, Marges de la philosophie.

«Seul celui qui n'est pas amoureux voit sa propre image dans l'eau », disait Rûmî. C'est l'au-delà du narcissisme, commente Sami-Ali ${ }^{1}$. Qu'est-ce à dire ? Que le regard frontal ne précipite point vers la mort et que la fontaine cesse d'être "si amère et si pleine de venin ${ }^{2}$ »? Que le miroir - dans une bouleversante et jouissive ascension telle que Diotime l'enseigne dans le Banquet ou dans une conversion vers soi-même dans la pure solitude de sa méditation, comme le stipule Plotin - ne reflète plus l'image de soi, mais celle de l'Autre, le Beau absolu ? Une solitude qui n'est point « isolement complaisant de l'individualisme " mais "recueillement ", selon l'expression de Maurice Blanchot ${ }^{3}$. Est-ce à cette seule condition que le sujet peut se regarder sans risquer la perte de soi ?

Quelles sont les assises pour qu'un tel regard soit possible ? Et à quelle véritable béance nous confronte le texte soufi ?

Houriya Abdelouahed, psychanalyste, maître de conférence à l'Université Paris 7. Denis Diderot, 83 rue Napoléon Fauveau, 95170 Deuil-la-Barre.

1. Sami-Ali, « Préface », Hallâj, Poèmes mystiques, trad. Sami-Ali, Sindbad, Paris, 1985.

2. Gilles de Lorris ; Jean de Meun, Le roman de la rose, Paris, Gallimard, coll. «Folio », p. 341.

3. M. Blanchot, L'espace littéraire, Paris, Gallimard, 1955, coll. «Folio», p. 13. 
La phrase de Rûmî contraste avec cette malheureuse conscience du jeune demoiseau de la fable ovidienne.

«Et bientôt, je briserais, baiser,

ce peu qui nous défend de l'extrême existence,

Cette tremblante, frêle, et pieuse distance Entre

moi-même et l'onde ${ }^{4}$. »

Pour avoir repoussé jeunes garçons et jeunes filles, Narcisse fut condamné à une passion et à une soif qu'il ne pouvait assouvir. Il mourut ainsi « au bord de son image » (Julia Kristeva). Sa punition, écrit Hadot, " répond à la fois à l'antique loi du Talion et à la logique de la faute ellemême. Le coupable est pris au mot. C'est l'application de la loi du Talion que réclame contre Narcisse l'amant tellement et longtemps méprisé ${ }^{5}$ ». Son châtiment est une "forme inouïe de démence », nous dit Ovide. Démence car Narcisse s'épuise, en fait, « à abolir une frontière, la frontière de la séparation entre soi et son image ${ }^{6} »$. S'il meurt victime de ses propres yeux, comme l'a dit Tirésias qui avait prédit son destin, $c^{\prime}$ est parce qu'il ignore « le principe de division dans l'espèce parlante ${ }^{7}$ ». Ce principe qui fait que le miroir est une « opération de division » (P. Legendre) et que le je est un autre ${ }^{8}$. À savoir l'existence d'un écart entre le je et ce qui se révèle comme image. Écart qui soutient la parole et notifie la négativité inhérente à l'ordre du langage à savoir le non collage du mot et de la chose, le vide constitutif de la relation signifiant/signifié. La barre. Le je est un autre parce que le miroir est une opération de division. Et le je est un autre pour celui qui accède à l'organisation représentative et à la catégorie de la pensée. Penser c'est « rendre l'altérité présente à soi ${ }^{9}$ ». En ce qui concerne cette conscience souffrante : «Que ne suis-je une présence non séparée ? »(P. Legendre), Valéry a, bien reconnu que l'instance spéculaire, loin de constituer le moi « en sa propriété, l'expro-

4. P. Valéry, Fragments de Narcisse, t. 1, p. 126-7-9, 13, cité par J. Derrida, Marges de la philosophie, Les éditions de Minuit, 1972, p. 340.

5. P.Hadot, « Le mythe de Narcisse et son interprétation par Plotin », dans NRP, Gallimard, 1976, coll. « Folio », p. 138.

6. P. Legendre, Dieu au miroir, Fayard, 1994, p. 44.

7. P. Legendre, op. cit., p. 44.

8. Sur cette phrase de Rimbaud, cf. J. Lacan, Le Séminaire, Livre II. Le moi dans la théorie de Freud et dans la technique de la psychanalyse, Le Seuil, 1978, p. 16. Lacan pointe la différence entre le je et l'instance imaginaire. Mais la question de la division sera affinée par P.Legendre, dans Dieu au miroir, op. cit., et L'inestimable objet de la transmission, Fayard, 1985. Cf. également H. Abdelouahed, " Des mots qui peignent ou la garantie de la ressemblance », dans La vie sensorielle, 2002. 9. R. Roussillon, Agonie, clivage, symbolisation, PUF, 2000, p. 238. 
prie aussitôt pour en brûler, stricto sensu, l'étape $10 »$. Aussi la démence de Narcisse consiste-t-elle précisément dans le fait qu'il ne se reconnaît pas. Et Tirésias disait que Narcisse ne pouvait vivre que s'il ne se voyait pas. L'on peut dire, à l'instar de P. Legendre, que Narcisse n'a pas accès au miroir car le miroir suppose cette division qui fait voir, selon la proposition hégélienne, l'origine comme résultat. Or, si la source fut pour Narcisse "si amère et si pleine de venin » $c^{\prime}$ est parce qu'elle n'a aucun profil pour elle-même, comme l'exprime J. Derrida qui continue : "C'est comme un regard absolu qui, toujours exorbité et jeté vers le visible, ne peut pas s'apercevoir lui-même et ne quitte jamais sa nuit ${ }^{11}$. " $\mathrm{N}^{\prime}$ ayant pas accès au miroir comme opération de division, la source, pour Narcisse, reste " origine pure ", "l'irréférence à soi 12 ». Aussi l'imaginaire s'y brise-t-il « plutôt qu'il ne s'y forme ${ }^{13}$ ». Or, pour quitter l'opacité de la nuit, pour advenir, il faut nécessairement accepter, intérioriser et faire sien l'écart, le vide ou la béance de l'altérité. Narcisse ignore une règle aussi simple que fondamentale : L'homme ne peut se rejoindre qu'en faisant un détour par l'autre. Éros est ce qui assure ce détour en donnant à la vue un statut privilégié dans l'expérience érotique. Chacun des partenaires servant à l'autre de miroir où dans l'œil de son vis-à-vis, c'est le reflet dédoublé de lui-même qu'il aperçoit et qu'il poursuit de son désir. Pour l'assouvir, il faut qu'il passe par l'autre. Or, Narcisse ne se sait « que comme voyant de cet objet, non comme regard de ce regard qui voit 14 ». Ce qui fait coïncider avec l'image du miroir, ce qui garantit la coïncidence, dans ce rapport de ressemblance est la béance de l'altérité. L'altérité est à la base d'une représentation de l'écart, la division d'avec soi. Écart entre le je et ce qui se révèle comme image. Écart qui notifie la perte.

Hadot, qui fait remarquer que Pausanias trouve invraisemblable qu'un homme capable d'aimer ne puisse distinguer entre un homme et un reflet, répond : « C'est que le trop rationaliste Pausanias ne comprend pas que Narcisse est saisi d'une démence dionysiaque ${ }^{15}$. »

Mais la phrase de Pausanias mérite qu'on continue cette réflexion sur la nécessité de l'autre pour que l'homme puisse se rejoindre. Un homme " capable d'aimer », dit Pausanias. C'est là l'intérêt du Phèdre qui met l'accent aussi bien sur l'amour que sur la vision.

10. J. Derrida, Marges de la philosophie, Les éditions de Minuit, 1972, p. 339.

11. Op. cit., p. 337.

12. J. Derrida, op. cit., p. 337.

13. Op. cit., p. 339.

14. P. Legendre, op. cit., p. 50 et p. 51.

15. P. Hadot, op. cit., p. 139. 
L'AMOUR DE SOI COMME OUVERTURE SUR L'AUTRE

Au texte de l'Alcibiade répond celui du Phèdre.

Socrate : "Quand nous regardons l'œil de quelqu'un qui est en face de nous, notre visage se réfléchit dans ce qu'on appelle la pupille, comme dans un miroir ; celui qui s'y regarde y voit son image.

Alcibiade : C'est exact.

Socrate : Ainsi, quand l'œil considère un autre œil, quand il fixe son regard sur la partie de cet œil qui est la plus excellente, celle qui voit, il s'y voit lui-même ${ }^{16}$. »

Le second texte non seulement fait écho mais introduit une dimension essentielle. "Il ne se rend pas compte, dit Socrate qui corrige les vues de Lysias, que dans son amant, ainsi qu'en un miroir, c'est lui-même qu'il aime ${ }^{17}$. »

De même, Ghazâlî (1059-1111) note : «L'homme ne cache pas qu'il s'aime soi-même, et ne cache pas qu'il n'aime autre que soi pour soi. S'imagine-t-il qu'il aime un autre que soi pour lui-même et non pour soi ? Voilà qui fait difficulté pour le faible d'esprit, si bien qu'il s'imagine inconcevable d'aimer un autre que soi pour lui-même ${ }^{18}$.» Et Ibn Arabi (1165-1240) de renchérir : « Personne n'aime un amant pour l'amant mais pour soi-même ${ }^{19}$. "

On pense que l'homme finit de s'aimer lorsqu'il se met à aimer son semblable. Or, l'être aimant s'aime aussi longtemps qu'il vit.

« Il n'en finira de s'aimer lui-même qu'au jour où il perdra la vie et non point au moment où il se mettra à aimer ses congénères, car il lui faudra au contraire continuer d'investir alors son propre moi fût ce en sourdine, pour pouvoir aimer ces êtres différents. Et l'on peut dire aussi qu'à partir de cette nouvelle origine c'est le moi lui-même qui vient à exister. Ce dont l'être n'est pas d'être perçu ou pensé, mais d'être aimé ${ }^{20}$. »

Cet amour constituera le cœur même de notre moi. Libido et moi sont du même côté. Le narcissisme est libidinal.

C'est le pont entre l'Alcibiade et le Phèdre. La visée amoureuse suppose l'insuffisance narcissique, l'abolition du plein, La reconnaissance du manque et le désir de le combler en passant par l'autre. Ne s'applique la loi du Talion

16. Platon, Alcibiade, CEuvres complètes, t. I, Les Belles lettres, 1970, 132e-133a.

17. Platon, Phèdre, CEuvres complètes, t. IV, Les Belles lettres, 1978, 255d.

18. Ghazâlî, Le livre de l'amour, trad. M.-L. Siauve, Vrin, 1986, p. 24.

19. Ibn Arabi, Al-Futûhât al-makkiyya, Dâr Sâdir (sans date d'édition), II, p. 333.

20. M. Dayan, La relation au réel dans la psychose, Paris, PUF, 1985, p. 131. 
qu'à celui qui n'a pas accès à la béance de l'altérité. Perte et séparation sont nécessaires pour qu'il y ait appel à l'autre. Cet appel ne figure point dans « le monologue " de Narcisse. Ses paroles n'ouvrent sur aucune extériorité, ne pointent vers aucune altérité. Dans « je m'aime ô reflet ironique de moimême » (P. Valéry) ou " c'est pour moi-même que je brûle d'amour » (Ovide), amour n'est pas ouverture sur l'autre. Amour n'est pas horizontal, comme dans le discours d'Aristophane, à savoir nostalgie de retrouvailles avec la moitié perdue. Amour n'opère pas non plus suivant un axe vertical qui porte vers l'immortalité, comme l'enseigne l'étrangère de Mantinée dans le Banquet. Dans « je m'aime » (je même) la forme est réflexive. Le miroir ne laisse pas voir l'origine comme résultat et le sujet parlant ne reçoit pas son message sous forme inversée.

Si aimer, comme nous le rappelle Christian David, c'est renaître ${ }^{21}$, l'on ne renaît qu'à travers l'autre. L'homme n'aime en effet que soi-même, mais... déplacé. "Quand l'homme aime la femme, il désire l'union, c'est-à-dire l'union la plus complète qui soit possible dans l'amour ; et dans la forme composée d'éléments, il n'existe pas d'union plus intense que celle de l'acte conjugal 22 », écrit Ibn Arabi. À la conscience malheureuse de Narcisse, est proposée ici une «synthèse » qui, engageant l'amant et l'aimé, permet cette " expression de l'aspiration sexuelle totale » où Freud voit le vrai sens de l'amour ${ }^{23}$.

Le ver de Rûmî répond, de façon admirable, à la phrase du prophète Mohammad (lâ yatamar'â ahadukum fíl mấ, ne vous mirez pas dans l'eau) répétant ainsi l'interdit pythagoricien.

En fait, semble dire Rûmî, le tabou pythagoricien - qui attribue au miroir un pouvoir maléfique - ne vaut que si l'on oublie que le regard ne précipita Narcisse dans les tréfonds de l'abîme que parce que le miroir n'était pas une opération de division et que la " misérable folie » (Ovide) abolit l'écart et la séparation et dénie la perte. Celui qui n'est pas pris dans les affres d'une « misérable folie », peut dire : « Tu vois un reflet dans le miroir. Est-ce voir véritablement ton visage 24 ? », ou encore : "Connais-tu quelqu'un qui ait vu son propre visage 25 ? » L'on peut, nous dit Rûmî, étancher sa soif sans risquer la perte de soi. "Ils dirent : Nous t'invitons à boire de l'eau, bois-en autant que tu veux 26 !»

21. C. David, L'état amoureux, PBP, 2002.

22. Ibn Arabi, Fusûs al-hikam, Beyrouth, éd. Dâr al-kitâb al-'arabî, 1946, t. I, p. 217 ; trad. T. Burckardt, La sagesse des prophètes, Albin Michel, 1974, p. 201.

23. Freud, Les trois essais sur la théorie de la sexualité, Gallimard, coll. "Idées », 1962.

24. Attâr, Le livre divin, Paris, Albin Michel, 1961, p. 406.

25. Attâr, op. cit., p. 407.

26. Rûmî, Rubâ'iyyât, trad. E. Meyerovitch, et J. Mortazavi, Albin Michel, 1987, p. 53. 
$C^{\prime}$ est à cette division que nous invite le texte soufi, en marge d'un discours théologique classique et dogmatique. Texte aux multiples sources. Texte pluriel. S'il intègre cette phrase de Muhammad (Dieu est Beau et aime la beauté), il ne fait pas sien l'interdit pythagoricien, repris par le prophète. Tout en puisant dans le Coran ou les hadîth-s (paroles du prophète Muham$\mathrm{mad}$ ), il dit son opposition ${ }^{27}$. Il puise également dans la théorie néoplatonicienne sans qu'il en soit un simple écho.

Plotin, en effet, réhabilite le miroir. Ce dernier n'est plus un lieu de perte et acquiert, au contraire, une dimension métaphysique puisque « le reflet originaire créateur du cosmos est un processus nécessaire et c'est seulement le reflet de ce reflet dans des substances périssables, qui nous éloigne de l'idéal et lui vaut en conséquence d'être condamné 28 ». Narcisse n'est condamné que parce qu'il s'éprend d'une beauté qui n'est que le reflet de la véritable beauté ${ }^{29}$. Narcisse est en deçà de ce mouvement qui permet de « voir la lune et non pas le reflet dans l'eau » (Rûmî).

Proche de la tradition néoplatonicienne, le soufi écrit : « Toi, tu n'es pas toi, tu es le reflet de Lui, aussi tu es beau et tu es bon [...] Ne contemple cette âme et ce corps. Contemple Sa Beauté et non la tienne ${ }^{30}$. "

Ce thème du reflet ou de l'univers phénoménal qui n'est que l'émanation de la Réalité ultime occupe une place considérable dans l'œuvre de Rûmî : " Tous les objets qui existent ici bas ne sont que des échantillons de l'autre monde ${ }^{31}$. » Ainsi, chez Rûmî, Narcisse n'a rien de narcissique. Narcisse est émerveillé, non de contempler sa propre beauté, mais parce qu'il admire le visage des jeunes mariés dans le jardin. « Non je me trompe, toimême et ces jeunes mariés, rectifie Rûmî qui s'adresse à Narcisse et aux jeunes mariés, et les fleurs, vous êtes émerveillés par mon Roi caché ${ }^{32}$. » Ainsi, les deux mouvements sont conjugués. La reconnaissance de l'incom-

27. "Que les minarets et les medresseh-s s'écroulent pour que le soufisme fleurisse », Rûmî, Rubâ'iyyât, trad. Halet Tchelebi A., Maisonneuve, Paris, 1984, p. 24.

28. J. Kristeva, Histoires d'amour, Paris, Denoël, p. 134.

29. «Que celui qui le peut aille donc et la suive (la beauté interne aux sanctuaires) jusque dans son intégrité ; qu'il abandonne la vision des yeux et ne se retourne pas vers l'éclat des corps qu'il admirait avant. Car si on voit les beautés corporelles, il ne faut pas courir à elles, mais savoir qu'elles sont des images, des traces et des ombres, et il faut s'enfuir vers cette beauté interne dont elles sont les images. Si on courait à elles pour les saisir comme si elles étaient réelles, on serait comme l'homme qui voulait saisir sa belle image portée sur les eaux (ainsi qu'une fable je crois le fait entendre), ayant plongé dans le profond courant, il disparut; il en est de même de celui qui s'attache à la beauté des corps et ne l'abandonne pas ; ce n'est pas son corps, mais son âme qui plongera dans ses profondeurs obscures et funestes à l'intelligence, il y suivra avec des ombres, aveugle séjournant dans l'Hadès " (Plotin, Ennéades, Les Belles lettres, 1960, I, 6, 8, 8).

30. Attar, Le livre divin, p. 406.

31. Rûmî, Le livre du dedans, trad. E. Meyerovitch, Sindbad, 1982, p. 262.

32. Rûmî, Rubâ'iyyât, p. 174. 
plétude qui fait la rencontre amoureuse et son dépassement pour le Beau absolu (rejoignant par là le discours de l'étrangère de Mantinée dans le Banquet de Platon). Mais le dépassement suppose que soit, nécessairement, assumée cette division du sujet.

Le texte soufi a le mérite de creuser une pensée de l'écart, du reflet et de l'image. Il ne s'agit pas seulement de l'écart, mais comment penser l'écart, pas seulement du reflet, mais d'une théorisation du reflet, non seulement de l'image, mais... d'une pensée sur l'image. Pensée qui connaîtra son point d'acmé avec Ibn Arabi qui aura le mérite de sortir l'image d'une thématique de l'eau et fera de la source autre chose qu'un jaillissement de cours d'eau.

\section{LE DESSIN DU SOUFFLE OU LA CONSTRUCTION DE L'IMAGE}

Quiconque lit Ibn Arabi est saisi par la puissance métaphorique et fictionnelle qui traverse son œuvre. Quiconque le lit est déconcerté non seulement par le foisonnement des thèmes, mais par la méthode, cette façon de déconstruire en permanence ce qu'il a déjà tracé, esquissé, dessiné. Comme le mouvement de l'air lui-même, ô combien présent, magnifiquement tracé par l'œuvre d'Ibn Arabi. Son mouvement est le mouvement de l'air même. Le mouvement caractérise sa pensée. Une pensée sur le mouvement, une pensée en mouvement. Aussi son texte défait-il et défie toutes les linéarités, à l'image du texte coranique ${ }^{33}$ et qui a le mérite de nous inviter à nous interroger sur ce qu'est un texte et comment lire un texte sans l'appauvrir et sans le réduire?

Si l'air a toujours été désigné comme support de la parole, Ibn Arabi introduit une dimension nouvelle en intégrant le mouvement de l'air dans sa définition du miroir. Dans le corpus arabo-musulman, c'est la première tentative de rassembler, dans un commentaire du texte coranique, le mouvement de l'air et la construction de l'image. Bien entendu, il ne perd pas de vue les acquis classiques tels : "Sans l'intermédiaire de l'autre, l'homme ne verrait pas sa véritable image » où le miroir ne sera pas uniquement du ressort de l'aliénation imaginaire, mais repose sur la dimension symbolique. Il ne fait pas fi, non plus, de la pensée mystique sur un cœur poli qui réfléchit la beauté divine tel un miroir. Laquelle beauté est recueillie selon la capacité visionnaire de chacun ${ }^{34}$. Mais il va encore plus loin.

33. «Lis! » est le premier verset coranique. Le texte coranique réfute tout positivisme historique et toutes les règles de la linéarité. La genèse devient le moment de déchiffrement.

34. Cf. H. Abdelouahed, La visualité du langage, Paris, L'Harmattan, 1998 et « Des noms qui regardent », dans Cliniques méditerranéennes, n 51/52, 1996. 
Outre le contexte intellectuel de l'Andalousie et l'héritage grec, Ibn Arabi se ressource aussi bien dans le texte coranique et prophétique que dans l'immense corpus linguistique et philologique arabe. En effet, le texte coranique est un texte arabe avec cette originalité grammaticale qui caractérise l'arabe comme langue sémitique. "Trilittéralité fixe des racines, écrit L. Massignon, syntaxe verbale relative à l'action et non à l'agent morphologie trivocalique (apprendre à vocaliser apprend à penser; la voyelle dynamise le texte consonantique amorphe et inerte) avec flexion unique pour les noms et les verbes [...]. Le mot arabe est composé d'un corps de consonnes seules écrites en noir sur la ligne, et d'une âme, leur vocalisation ${ }^{35}$. » Et c'est entre une pensée qui pose Dieu comme «l'Autre causal» (P. Legendre) et la langue liturgique de l'islam qu'on peut situer un certain axe de la pensée d'Ibn Arabi. Ainsi lorsqu'il réfléchit sur le verset : "Quand je l'aurai façonné et insufflé en lui de mon Esprit », dit le Coran au sujet d'Adam (Cor. $15: 29 ; 38$ : 72), Ibn Arabi, s'écartant du savoir théologique classique, interprète le verset ainsi :

"Ceci concerne la venue des voyelles sur les lettres après le façonnement de ces dernières. Elles réapparaissent alors dans une "autre constitution" appelée mot (kalima), tout comme un individu de notre espèce se nomme "homme". C'est de cette manière que se constitua le monde des mots (kalimât) et des vocables (alfâz) à partir du monde des lettresconsonnes ${ }^{36}$. »

«Les lettres, poursuit-il, sont les éléments matériels (mawâd) des mots, comme l'eau, la terre, le feu et l'air pour la constitution de nos corps. »

Si la religion joue comme " garant de la Raison en ce qu'elle offre au sujet l'arrêt de la régression à l'infini dans la causalité [...] l'Autre originel devient l'Autre causal 37 », il n'en demeure pas moins que c'est le « le dieu de l'instant » dans un perpétuel mouvement car porté par le souffle défait par l'air qui vient s'inscrire dans le corpus akbarien (celui d'Ibn Arabi). Et c'est ainsi que nous pouvons lire:

"Lorsque Dieu voulut voir les essences ( $\left.a^{\prime} y a ̂ n\right)$ de Ses Noms les plus beaux et tu peux également dire : Dieu voulut voir Sa propre essence en un objet (... ) Or, la vision qu'a l'être de lui-même n'est pas pareille à

35. L. Massigon, «L'arabe, langue liturgique de l'Islam », dans L'Islam et l'Occident, 1947, p. 160. 36. Ibn Arabi, Fut., I., p. 84-85 ; trad. D. Gril, « La science des lettres », dans Les illuminations de la Mecque, p. 482.

37. P. Legendre, Dieu au miroir, p. 64. 
celle que lui procure une autre réalité dont il se sert comme d'un miroir ${ }^{38}$. »

Il n'est que Ses noms. Ses noms ne sont autres que Lui (" famâ thamma illlâ asmâ'uh wa laysa siwâhu 39 »). Si le texte coranique annonce : "Il est l'Un », Ibn Arabi précise : "Il est l'Un le Multiple ». Et lorsque le Coran annonce : "Lorsqu'il veut une chose, il lui dit: "sois !" et la chose est » (Cor. $2: 17$; 3 : $47 ; 3: 59 \ldots)$, Ibn Arabi coupe, découpe le verset, introduit sa propre parole. «Ton regard est l'équivalent de Sa parole : lorsque nous voulons une chose, nous disons. C'est l'équivalent de ce qu'on entend par regard. Nous lui disons : "sois!" C'est l'équivalent du regard. Et "elle est". C'est l'équivalent de l'image ${ }^{40}$.» Portée par la force propulsive du désir, la parole émane de cet Un Multiple. Et c'est grâce au miroir que la multiplicité vit le jour. Mais la multiplicité, n'est pas celle de l'Intelligence et de l'intelligible comme dans le système plotinien. Elle est celle des noms. En outre, la procession telle qu'elle figure chez le philosophe alexandrin est récusée. La multiplicité des noms naît « d'un seul coup ${ }^{41}$ ». Le miroir devient, à ce moment, hadrat al-imkân (la Présence des possibles). Quant à l'image, elle n'est autre que toi selon ta prédisposition ou ta capacité, « soit astre, soit homme, ou encore cheval 42 ».

" Notre ordre est une seule parole, il est prompt comme un clin d'œil », dit le texte coranique (Cor. 54 : 50). La rapidité est telle que personne ne connaît le pouvoir dont il s'agit, commente Ibn Arabi, car " l'homme ne se rend pas spontanément compte de ce qu'il n'est pas et ce qu'il est à chaque souffle » (fî̀ kulli nafasin lâ yakûnu thumma yakûn) ${ }^{43}$. L'image est portée par le souffle. Et ce dernier « correspond à l'élément air (an-nafas munâsib li 'unsur al-hawấ, ${ }^{44}$.»

L'air est l'invisible support de l'image. Cette image qui entendit l'impératif : «sois ! » Impératif comme traversée originaire de la parole et comme ouverture sur le corps de la langue. Impératif comme métaphore de l'origine. Et l'image, entendant la voix et consentant à venir, inscrit, dans la venue au monde, le désir de l'Autre. Il dit : "Sois ! " Et la chose est. C'est l'air de phôné qui trace l'image. P. Fédida note justement que l'air phonique " est le seul support de l'image visuelle ${ }^{45}$ ». L'air phonique du mot dessine l'image qui

\footnotetext{
38. Ibn Arabi, Fus., I, p. 48 ; trad., p. 21.

39. Ibn Arabi, Fut., IV, p. 403.

40. Ibn Arabi, Fut., IV, p. 316.

41. Ibn Arabi, al-thadhbîrât al-ilâhiyya, éd. Neyberg, Leiden, 1919, p. 121.

42. Emir Abdel-Kader, Kitâb al-mawâaif, éd. Dâr al-yaqaza al-'arabiyya, Damas, 1967, p.1345.

43. Fus., I, p. 155 ; trad., p. 152.

44. Fut., II, p. 123.

45. P. Fédida, Le site de l'étranger, PUF, 1995, p. 66.
} 
prend tel ou tel aspect : astre, homme ou encore cheval. C'est la voix disant, c'est le vocable du mot qui produit l'image.

Ces images disent : "Kunnâ mabtûnîna fìhi, nous étions en Lui, nous étions emmagasinées en Lui, ou enveloppées en Lui ${ }^{46}$. » Ce qui vient du silence de la nuit s'efface avec l'aurore de la parole. Aussi le verset coranique commence-t-il par al-bâri' (le Créateur) et termine par al-musawwir (Celui qui donne la forme ou l'aspect). Le stade du taswîr étant le dernier ${ }^{47}$. L'aspect, dit Henri Maldiney, est la première dimension du verbe ${ }^{48}$. « Il a commencé par la parole. Il nous a ainsi créés en disant : "sois ! " La première qualité que nous ayons eue à son égard fut l'écoute " (fabada'a subhânahu bi-al-kalâm fa khalaqanâ faquâla kun). Il nous a créés grâce à la parole, il dit : " kun ! (sois !) " 49 ». "Sans la parole, nous serions aujourd'hui dans un néant. Mais il dit : "Sois" ». Ainsi, " Il fut le premier locuteur et je fus le premier auditeur ${ }^{50}$. » L'altérité travaillait déjà la voix. Et avec elle, la discontinuité et la coupure.

" “Sois !" est une particule d'existence car n'émane d'elle que l'existence ${ }^{51}$. » Aussi « al-dhikr wujûd, la parole est-elle ce qui fait exister ${ }^{52}$ ».

La rapidité est telle et que l'être n'est pas et est à chaque souffle. Cette phrase d'Ibn Arabi, Ja'far al-Sâdiq lui donne un autre ton : « Il apparaît dans Ses paroles, mais vous ne Le voyez pas. » La seule visibilité de l'image est ce que trace le souffle disant. Il est ainsi le voilé-dévoilé ${ }^{53}$. Et ce sont justement ces multiples manifestations, à l'infini, qui constituent un voile.

$\mathrm{Si}$, dans la pensée mystique classique, le voile constitue un écran, un obstacle à la vision, « mais aussi une protection contre le feu dévorant de l'amour divin ${ }^{54}$ », comme le dit E. Meyerovitch, chez Ibn Arabi, les lettres constituent un voile. La multiplicité des noms est également un voile... Raison pour laquelle, il écrit " fa irfa" annî al-labs al-ladhî yahûlu bayni wa bayna al-khalq aljadîd, Supprime cette illusion qui m'empêche de saisir la création récurrente ». Ainsi, " il n'y a de transfert que d'un voile à un autre, car la manifestation divine ne se répète jamais deux fois. La multiplicité des images

46. Littéralement: dans son ventre.

47. «Il est Dieu! le Créateur; Celui qui donne un commencement à toute chose ; Celui qui façonne. Les noms les plus beaux Lui appartiennent » (Coran 59 :24). Cf. Fut., IV, p. 213.

48. Maldiney H., Aîtres de la langue et demeures de la pensée, Éd. L'Âge d'Homme, Lausanne, 1975, p. 14.

49. Fut., I, p. 321.

50. Fut., III, p. 567.

51. Fut., II, p. 280-281.

52. Fut., IV, p. 167.

53. Fut., III, p. 215.

54. E. Meyerovich, Mystique et poésie en Islam, Djalâl ud-Dîn Rûmî et l'ordre des derviches tourneurs, Belgique, Desclée de Brouer, p. 205. 
est nécessaire 55 ». Et « wa inshâ'u al-suwar lâ yatanâha, (la création des images est constante) ${ }^{56} »$.

Si le sculpteur devant le bloc de marbre informe, compact et solide s'interroge sur l'image qui va sortir de la matière dense et informe : «Qu'en fera mon ciseau ? Sera-t-il Dieu, table ou cuvette ${ }^{57}$ ? » Le ciseau va tailler, couper et l'instrument guide la main vers « la genèse de l'image », écrit L. Marin. Ici, $c^{\prime}$ est l'air phonique qui va couper, sortir de la masse dense et indistincte l'image qui ne sera autre que le nom : astre, homme ou encore cheval, dieu, table ou cuvette. Un ruissellement de noms qui rejette ou récuse la pensée classique d'une création qui débuterait avec un commencement et qui serait maintenant achevée. Dans le système akbarien "walâ tazâlu kun walâ yazâlu al-takwîn. Le "sois !" est permanent. Aussi la création est-elle récurrente 58 ». Quel est le commencement et quelle est la fin ? La création est récurrente, éternellement nouvelle car « co-naissante à un perpétuel départ 59 » qui est celle de la langue dans l'acte de nomination ». Un éternel commencement qui fait que l'homme, selon la belle expression de Maldiney, devient « contemporain de son origine 60 ».

Astre, homme ou encore cheval, ou ruisseau, ou encore rivière. Et pourquoi pas source ? Seul l'air de la parole dessine l'image, rapide comme un clin d'œil, mais discontinue et traversée par l'altérité. Et seul le « tournant du souffle» (P. Celan) dit la source.

Lorsque la voix dit la source, lorsque la source devient parole, lorsque la parole de l'interprète se situe dans un écart par rapport à une littéralité écrasante des ressources mytho-poétiques dont le texte est porteur, quant l'interprète s'ouvre sur le dehors, l'extériorité, c'est la pensée qui se déploie et qui se dit. Se ressourcer sert à dire l'écart par rapport à la source. Tel est le propre de la pensée.

\section{BIBLIOGRAPHIE}

AbDel-Kader, (l'Emir). 1967. Kitâb al-mawâqif, éd. Dâr al-yaqaza al-'arabiyya, Damas. Abdelouahed, H. 1998. La visualité du langage, Paris, L'Harmattan.

Abdelouahed, H. 1996. "Des noms qui regardent ", Cliniques méditerranéennes, $\mathrm{n}^{\circ} 51 / 52$, p. $145-155$.

55. Fut., IV, p. 105.

56. Fut., IV, p. 214.

57. Cf. L. Marin, « Trois gloses », dans NRP, $\mathrm{n}^{\circ}$ 44, automne 1991, p. 129.

58. Fut., II, p. 385.

59. H. Maldiney, Aîtres de la langue et demeures de la pensée, L'Âge d'Homme, Lausanne, p. 94. 60. Ibid. 
Abdelouahed, H. 2002. " Des mots qui peignent ou la garantie de la ressemblance », dans La vie sensorielle, Paris, PUF.

ATtÂR, 1961. Le livre divin, Paris, Albin Michel.

Blanchot, M. 1955. L'espace littéraire, Gallimard, Paris, coll. « Folio ».

DAYAN, M. 1985. La relation au réel dans la psychose, Paris, PUF.

DAVID, C. 2002. L'état amoureux, PBP.

DerridA, J. 1972. Marges de la philosophie, Les éditions de Minuit.

FÉDIDA, P. 1995. Le site de l'étranger, Paris, PUF.

FREUD, S. 1962. Les trois essais sur la théorie de la sexualité, Gallimard, coll. « Idées ».

GHAZÂLî, 1986. Le livre de l'amour, trad., M.-L. Siauve, Vrin.

HADOT, P. 1976. « Le mythe de Narcisse et son interprétation par Plotin », NRP, Gallimard, coll. « Folio », p. 127-160.

IBN ARABI. Al-Futûhât al-makkiyya, Dâr Sâdir (sans date d'édition) 4 tomes.

IBN ARABI. 1946. Fusûs al-hikam, Beyrouth, éd. Dâr al-kitâb al-'arabî, t. I, trad. T. Burckardt, La sagesse des prophètes, Albin Michel, 1974, p. 201.

IBN ARABI. 1919. Al-thadhbîrât al-ilâhiyya, Éd. Neyberg, Leiden.

Kristeva, J. Histoires d'amour, Denoël, p. 134, coll. " Folio ».

LACAN, J. 1978. Le Séminaire, Livre II. Le moi dans la théorie de Freud et dans la technique de la psychanalyse, Le Seuil.

LEGENDRE, P. 1994. Dieu au miroir, Fayard.

LEGENDRE, P. 1985. L'inestimable objet de la transmission, Fayard.

LORRIS, G. de. ; Meun, J. de. Le roman de la rose, Paris, Gallimard, coll. « Folio ».

Maldiney, H. 1975. Aîtres de la langue et demeures de la pensée, éd. L'Age d'Homme, Lausanne.

MARIN, L. 1991. «Trois gloses », in NRP, $\mathrm{n}^{\circ} 44$, automne, p. 129-150.

MAssigon, L. 1947. "L'arabe, langue liturgique de l'Islam », dans L'Islam et l'Occident, Rivages, Les cahiers du Sud, 1947.

Meyerovich, E. Mystique et poésie en Islam, Djalâl ud-Dîn Rûmî et l'ordre des derviches tourneurs, Belgique, Desclée de Brouer.

Platon. 1970. Alcibiade, CEuvres complètes, tome I, Les Belles lettres.

Platon. 1978. Phèdre, CEuvres complètes, tome IV, Les Belles lettres.

Plotin. 1960. Ennéades, Les Belles lettres, I.

Roussillon, R. 2000. Agonie, clivage, symbolisation, Paris, PuF.

RûMî. 1982. Le livre du dedans, trad. Meyerovitch E., Sindbad.

RÛMî. 1987. Rubâ'iyyât, trad. Meyerovitch E., et Mortazavi J., Albin Michel.

RÛMî, 1984. Rubâ'iyyât, trad. Halet Tchelebi A., Maisonneuve, Paris.

SAmi-Ali, 1985. « Préface », Hallâj, Poèmes mystiques, trad. Sami-Ali, Sindbad, Paris.

Résumé

À côté du corpus théologique classique, le texte soufi définit le miroir comme opération de division. Division entre le je et ce qui se présente comme image.

Mais cette pensée de l'image connaîtra son point d'acmé sous la plume d'Ibn Arabi qui l'arrachera à une phénoménologie de l'eau en liant l'air de phôné à la construction de l'image. 
Ainsi, se dit l'écart aussi bien par rapport à une lecture littérale du texte qu'à l'héritage grec et à la pensée mystique elle-même.

Ibn Arabi ouvre sur une extériorité permettant à la pensée de se déployer et de se dire.

Mots clés

Source, image, miroir, altérité, air de la parole.

THE SOURCE AND DIFFERENCE

Summary

Alongside the classical theological corpus of works, the Sufi text defines the mirror as an operation of division. Division between the " $\mathrm{I}$ » and what comes up as an image. But this thinking of the image will reach its highest point under the pen of Ibn'Arabi who was to tear it away from a phenomenology of water and link it to the air of phone, to the construction of the image.

So difference is expressed both in relation to a literal reading of the Text and to the Greek heritage and mystical thought itself.

Ibn'Arabi opens out onto an exteriority that allows thought to fold out and find expression.

Keywords

Source, image, mirror, alterity, air of speech. 